USM-TH-74

\title{
The Quark Orbital Angular Momentum in a Light-Cone Representation
}

\author{
Bo-Qiang $\mathrm{Ma}^{a}$ and Ivan Schmidt ${ }^{b}$ \\ ${ }^{a}$ CCAST (World Laboratory), P.O. Box 8730, Beijing 100080, China \\ and Institute of High Energy Physics, Academia Sinica, P. O. Box 918(4), \\ Beijing 100039, China \\ Email: mabq@bepc3.ihep.ac.cn \\ ${ }^{b}$ Departamento de Física, Universidad Técnica Federico Santa María, \\ Casilla 110-V, Valparaíso, Chile \\ Email: ischmidt@fis.utfsm.cl
}

\begin{abstract}
We perform an analysis of the quark angular momentum in a light-cone representation by taking into account the effect due to the Melosh-Wigner rotation and find that there is a relativistic correction factor connecting the quark orbital angular momentum with the quark model spin distribution: $L_{q}(x)=$ $<M_{L}(x)>\Delta q_{Q M}(x)$. The quark orbital angular momentum $L_{q}(x)$ and the quark helicity distribution $\Delta q(x)$ are connected to the quark model spin distribution $\Delta q_{Q M}(x)$ by a relation: $\frac{1}{2} \Delta q(x)+L_{q}(x)=\frac{1}{2} \Delta q_{Q M}(x)$, which means that one can decompose the quark model spin contribution $\Delta q_{Q M}(x)$ by a quark helicity term $\Delta q(x)$ plus an orbital angular momentum term $L_{q}(x)$. There is also a new relation connecting the quark orbital angular momentum with the measurable quark helicity distribution and transversity distribution $(\delta q(x))$ : $\Delta q(x)+L_{q}(x)=\delta q(x)$, from which we may have new sum rules connecting the quark orbital angular momentum with the nucleon axial and tensor charges.
\end{abstract}

PACS numbers: 11.55.Hx, 13.60.Hb, 13.88.+e, 14.20.Dh 


\section{Introduction}

The proton spin problem has received attention for about a decade since the observation of a small value of the integrated spin structure function for the proton in the experiment of polarized deep inelastic scattering (DIS) of leptons on the proton by the European Muon Collaboration [1]. The experimental results suggested a very small quark helicity sum which is far from 1 , the value of the quark spin contribution to the proton spin in the quark model. This triggered the proton "spin crisis" or "spin puzzle" which implied the conflict between the experimental results and the quark model, and there have been thousands of papers related to various aspects on this issue [2]. The prevailing viewpoint seems to be that the proton spin structure is in conflict with the quark model.

However, this viewpoint should be changed from a number of investigations [3] [4, 5, 6, [] on the proton spin structure in the light-cone formalism, which is the most convenient framework to describe deep inelastic scattering (DIS). It has been

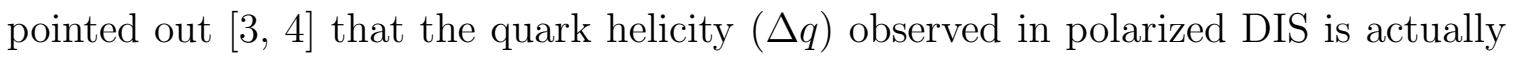
the quark spin defined in the light-cone formalism and it is different from the quark spin $\left(\Delta q_{Q M}\right)$ as defined in the quark model. Thus the small quark helicity sum observed in polarized DIS is not necessarily in contradiction with the quark model in which the proton spin is provided by the valence quarks [5, 7]. There have been studies which support the above physical results in different frameworks, such as in a conventional QCD Lagrangian based formalism [B] and in a quark model approach in the rest reference frame [9].

In this paper we will perform a light-cone analysis of the quark orbital angular momentum along with the developments in Refs. [3, [4, [6] by taking into account the effect due to the Melosh-Wigner rotation [10] which is an important ingredient in the light-cone formalism [11]. The light-cone formalism is suitable to describe the relativistic many-body problem and there have been many successful applications of the light-cone quark model to various physical processes [12]. The effects due to the Melosh-Wigner rotation have been calculated for the nucleon axial charges [3, [4 and magnetic moments [4], and most recently for the nucleon tensor charges 
[6]. It will be shown that there is also a Melosh-Wigner rotation factor connecting the quark orbital angular momentum with the quark model spin distribution: $L_{q}(x)=<M_{L}(x)>\Delta q_{Q M}(x)$, in analogy to the quark helicity distribution [3, 团 and transversity distribution [6]. The quark orbital angular momentum $L_{q}(x)$ and the quark helicity distribution $\Delta q(x)$ are connected to the quark model spin distribution $\Delta q_{Q M}(x)$ by a relation: $\frac{1}{2} \Delta q(x)+L_{q}(x)=\frac{1}{2} \Delta q_{Q M}(x)$, which means that one can decompose the quark model spin contribution $\Delta q_{Q M}(x)$ by a quark helicity term $\Delta q(x)$ plus an orbital angular momentum term $L_{q}(x)$. We have also a new relation connecting the quark orbital angular momentum with the measurable quark helicity distribution and transversity distribution: $\Delta q(x)+L_{q}(x)=\delta q(x)$, from which we may have new sum rules connecting the quark orbital angular momentum with the nucleon axial and tensor charges.

\section{The Melosh-Wigner rotation in a light-cone rep- resentation}

The light-cone formalism provides a convenient framework for the relativistic description of hadrons in terms of quark and gluon degrees of freedom [13]. Light-cone quantization has a number of unique features that make it appealing, most notably, the ground state of the free theory is also a ground state of the full theory, and the Fock expansion constructed on this vacuum state provides a complete relativistic many-particle basis for diagonalizing the full theory [4. As we have known, it is proper to describe deep inelastic scattering as the sum of incoherent scatterings of the incident lepton on the partons in the infinite momentum frame or in the light-cone formalism. The Melosh-Wigner rotation [10] is one of the most important ingredients of the light-cone formalism, and it relates the light-cone (LC) spin state wavefunctions

$q_{L C}^{\uparrow, \downarrow}$ to the ordinary instant-form (IF) spin state wavefunctions $q_{I F}^{\uparrow, \downarrow}$ by the equation:

$$
\begin{aligned}
q_{L C}^{\uparrow} & =w\left[\left(k^{+}+m\right) q_{I F}^{\uparrow}+k^{R} q_{I F}^{\downarrow}\right] ; \\
q_{L C}^{\downarrow} & =w\left[-k^{L} q_{I F}^{\uparrow}+\left(k^{+}+m\right) q_{I F}^{\downarrow}\right],
\end{aligned}
$$


where $w=\left[\left(k^{+}+m\right)^{2}+k_{\perp}^{2}\right]^{-1 / 2}, k^{R, L}=k^{1} \pm i k^{2}$, and $k^{+}=k^{0}+k^{3}=x \mathcal{M}$ in which the light-cone invariant mass $\mathcal{M}$ of the many-body system is given by $\mathcal{M}^{2}=\sum_{q} \frac{m_{q}^{2}+k_{q \perp}^{2}}{x_{q}}$. In a weak-binding limit one can approximate this rotation by putting $\mathcal{M}=M_{B}$, but in our case the rotation applies to every individual spin state of a whole relativistic many-body system, therefore the rotation Eq. (11) should not be confused as a single rotation for a single free particle.

We express the instant-form spin state wavefunctions by the 4-dimensional vectors $q_{I F}^{\uparrow}=(1,0,0,0)$ and $q_{I F}^{\downarrow}=(0,1,0,0)$, therefore we can write the light-cone spin state wavefunctions as

$$
\begin{aligned}
& q_{L C}^{\uparrow}=\left(w\left(k^{+}+m\right), w k^{R}, 0,0\right) ; \\
& q_{L C}^{\downarrow}=\left(-w k^{L}, w\left(k^{+}+m\right), 0,0\right) .
\end{aligned}
$$

Then we can write the light-cone spin space wave function of a bound state in terms of direct products of the above light-cone spin state wavefunctions of the individual quarks, with the many-body system keeps the same spin structure as in the ordinary rest reference frame. In case we calculate a certain matrix element of a physical quantity with known operator, we can study the effect in the spin sector by acting the operator directly on the corresponding light-cone spin state wavefunctions which is one part of the whole light-cone wavefunction for the bound system.

Now we reanalyse the quark helicity distribution and transversity distribution in the light-cone representation sketched out above. The quark helicity distribution is defined by the axial current matrix element

$$
\Delta q=<p, \uparrow\left|\bar{q} \gamma^{+} \gamma_{5} q\right| p, \uparrow>
$$

We now perform the operator $\gamma^{+} \gamma_{5}$ directly on the spin space part of the light-cone wavefunction for a bound system and the actions from the spin sector are

$$
\begin{array}{lcc}
\bar{q}_{L C}^{\uparrow} \gamma^{+} \gamma_{5} q_{L C}^{\uparrow} & = & M_{q}\left(x, k_{\perp}\right) ; \\
\bar{q}_{L C}^{\downarrow} \gamma^{+} \gamma_{5} q_{L C}^{\uparrow} & = & 0 ; \\
\bar{q}_{L C}^{\uparrow} \gamma^{+} \gamma_{5} q_{L C}^{\downarrow}= & 0 ; \\
\bar{q}_{L C}^{\downarrow} \gamma^{+} \gamma_{5} q_{L C}^{\downarrow}= & =-M_{q}\left(x, k_{\perp}\right),
\end{array}
$$

where

$$
M_{q}\left(x, k_{\perp}\right)=\frac{\left(k^{+}+m\right)^{2}-k_{\perp}^{2}}{\left(k^{+}+m\right)^{2}+k_{\perp}^{2}}
$$


is the Melosh-Wigner rotation factor due to the quark intrinsic transverse motions, as derived in Ref.[3, 4]. Thus we have

$$
\Delta q(x)=\int\left[d^{2} k_{\perp}\right] M_{q}\left(x, k_{\perp}\right) \Delta q_{Q M}\left(x, k_{\perp}\right)=<M_{q}(x)>\Delta q_{Q M}(x),
$$

where $\Delta q_{Q M}$ is the quark spin distribution in the quark model.

In analogy, we can perform a similar analysis of the quark transversity distribution which is defined by the tensor current

$$
<p, s\left|\bar{q} \sigma^{\mu \nu} q\right| p, s>=\delta q \bar{U}(p, s) \sigma^{\mu \nu} U(p, s)
$$

where $U(p, s)$ is the Dirac spinor of a free nucleon with momentum $p$ and polarization vector $s$. Thus we can calculate the quark transversity distribution from the $1+$ component

$$
\delta q=i<p, \downarrow\left|\bar{q} \sigma^{1+} q\right| p, \uparrow>
$$

The actions of the operator $\sigma^{1+}$ on the spin space part of the light-cone wavefunction are found to be

$$
\begin{array}{rcc}
\bar{q}_{L C}^{\uparrow} \sigma^{1+} q_{L C}^{\uparrow} & = & -2 w^{2} k_{2}\left(k^{+}+m\right) ; \\
\bar{q}_{L C}^{\downarrow} \sigma^{1+} q_{L C}^{\uparrow} & = & -i \widetilde{M}_{q}\left(x, k_{\perp}\right)-i w^{2}(k 1+i k 2)^{2} ; \\
\bar{q}_{L C}^{\uparrow} \sigma^{1+} q_{L C}^{\downarrow} & = & i \widetilde{M}_{q}\left(x, k_{\perp}\right)+i w^{2}(k 1-i k 2)^{2} ; \\
\bar{q}_{L C}^{\downarrow} \sigma^{1+} q_{L C}^{\downarrow} & = & 2 w^{2} k_{2}\left(k^{+}+m\right),
\end{array}
$$

where

$$
\widetilde{M}_{q}\left(x, k_{\perp}\right)=\frac{\left(k^{+}+m\right)^{2}}{\left(k^{+}+m\right)^{2}+k_{\perp}^{2}}
$$

is the Melosh-Wigner rotation factor found in Ref. [6]. One easily finds that the other additional terms in Eq. (9) vanish upon integration over the azimuth of $k_{\perp}$, thus we have

$$
\delta q(x)=\int\left[d^{2} k_{\perp}\right] \widetilde{M}_{q}\left(x, k_{\perp}\right) \Delta q_{Q M}\left(x, k_{\perp}\right)=<\widetilde{M}_{q}(x)>\Delta q_{Q M}(x)
$$

which was first given in Ref.[6]. It is found that there is a relation [6]: $1+M_{q}=2 \widetilde{M}_{q}$, from which a relation connecting the quark helicity and transversity distributions to the quark model spin distribution was suggested [7]

$$
\Delta q_{Q M}(x)+\Delta q(x)=2 \delta q(x)
$$


This relation was first proposed in Ref. [7] and the consequences from it's application have also been discussed [7, 15]. There has been recently a proof of this relation in a QCD Lagrangian based formalism [8].

We proved in the above the efficiency to derive the known effects due to the Melosh-Wigner rotation in the quark helicity and transversity distributions [3, 4, 6] in a simple representation with the light-cone spin state wavefunctions Eq.(2). As we have pointed out, we can easily apply a known operator on those spin state wavefunctions directly to study the relativistic effect in the spin sector of the quark model. Now we make an analysis of the quark orbital angular momentum in this light-cone representation. The orbital angular momentum is defined by

$$
L_{q}=-i k \times \nabla_{k}
$$

and the contribution in the proton spin direction can be calculated from the operator

$$
\hat{L}_{q}=-i\left(k_{1} \frac{\partial}{\partial k_{2}}-k_{2} \frac{\partial}{\partial k_{1}}\right)
$$

The actions of the operator $\hat{L}_{q}$ on the individual spin space part of the light-cone wavefunction are found to be

$$
\begin{array}{llc}
\bar{q}_{L C}^{\uparrow} \hat{L}_{q} q_{L C}^{\uparrow} & = & M_{L}\left(x, k_{\perp}\right) ; \\
\bar{q}_{L C}^{\downarrow} \hat{L}_{q} q_{L C}^{\uparrow} & = & w^{2}\left(k_{1}+i k_{2}\right)\left(k^{+}+m\right) ; \\
\bar{q}_{L C}^{\uparrow} \hat{L}_{q} q_{L C}^{\downarrow} & = & w^{2}\left(k_{1}-i k_{2}\right)\left(k^{+}+m\right) ; \\
\bar{q}_{L C}^{\downarrow} \hat{L}_{q} q_{L C}^{\downarrow} & = & -M_{L}\left(x, k_{\perp}\right),
\end{array}
$$

where

$$
M_{L}\left(x, k_{\perp}\right)=\frac{k_{\perp}^{2}}{\left(k^{+}+m\right)^{2}+k_{\perp}^{2}}
$$

is a new Melosh-Wigner rotation factor. The other terms besides $M_{L}\left(x, k_{\perp}\right)$ in Eq. (15) vanish upon integration over the azimuth of $k_{\perp}$, thus we have the quark orbital angular momentum

$$
L_{q}(x)=\int\left[d^{2} k_{\perp}\right] M_{L}\left(x, k_{\perp}\right) \Delta q_{Q M}\left(x, k_{\perp}\right)=<M_{L}(x)>\Delta q_{Q M}(x)
$$

which connects the quark orbital angular momentum to the quark model spin distribution by a Melosh-Wigner rotation. 
It is interesting to notice that

$$
\frac{1}{2} M_{q}+M_{L}=\frac{1}{2}
$$

from which we have a relation

$$
\frac{1}{2} \Delta q(x)+L_{q}(x)=\frac{1}{2} \Delta q_{Q M}(x),
$$

which means that we can decompose the quark model spin distributions $\frac{1}{2} \Delta q_{Q M}(x)$ into a quark helicity term $\frac{1}{2} \Delta q(x)$ plus an orbital angular momentum term $L_{q}(x)$. Thus from a relativistic viewpoint the orbital angular momentum is non-zero even for an s-wave quark in the quark model. This confirms the statement [3] that the angular momentum contribution from an s-wave quark to the proton spin should be equal to the quark model spin $\frac{1}{2} \Delta q_{Q M}$, but not the quark helicity $\frac{1}{2} \Delta q(x)$. Thus the small quark helicity sum $\Delta \Sigma=\sum_{q} \Delta q(x)$ observed in polarized DIS is not in conflict with the quark model. A reduction in the quark helicity is complemented by an increase in the orbital angular momentum from a relativistic viewpoint. This also explains why our result is in fact not in conflict with the conventional statement that quark spin carries only a small part of the proton spin if one takes $\frac{1}{2} \Delta q$ as the "quark spin". However, the angular momentum contribution from the quark to the proton spin should be the "quark spin" plus the relativistic orbital angular momentum, which is actually the quark model spin $\frac{1}{2} \Delta q_{Q M}$. From this sense, the quark orbital angular momentum plays an important role in the spin content of the nucleon. In fact, the importance of the quark orbital angular momentum in the nucleon spin structure was originally noticed by Sehgal [16], and some other relevant aspects are also under discussion [17.

The above results from the Melosh-Wigner rotation are valid in a quite general framework of the light-cone quark model 4, 12] which is in fact non-perturbative. We point out that the relation Eq.(19) is also valid in different approaches such as in the bag model or if one calculates the matrix elements of the quark helicity and orbital angular momentum distributions in the nucleon rest frame with the ordinary free Dirac spinors. Thus this relation might be considered as a rather model independent relation with general physical implications, similar to the known relation Eq.(12) 
proposed in Ref.[7]. Even if one takes both Eqs.(12) and (19) as model dependent results, one may still make independent measurements of the model quantity $\Delta q_{Q M}(x)$ with either Eq.(12) or Eq.(19) independently, thus testing the validity of the model results. We notice that the Melosh-Wigner rotation factors satisfy a relation

$$
M_{q}+M_{L}=\widetilde{M}_{q}
$$

thus we have

$$
\Delta q(x)+L_{q}(x)=\delta q(x),
$$

which is a new and elegant relation directly connecting the three measurable quantities $\Delta q(x), \delta q(x)$, and $L_{q}(x)$.

At this point it is important to clarify the range of validity of the particular relativistic bound-state model we are using. It is a three-quark valence model, which therefore does not consider higher Fock states, and which can then be considered as a starting point for dynamically generated gluon and sea distributions 18. But to the extent that gluons generate the binding, it does contain intrinsic gluons [19]. So we expect this model to be valid at least up to a momentum scale $Q^{2}=Q_{0}^{2}$, where $Q_{0}$ can be taken as the color inverse neutral target size, approximately $1 \mathrm{GeV}^{2}$. In practice, the light-cone quark model has been successfully applied to processes with higher $Q^{2}$ of about a few $\mathrm{GeV}^{2}$ [12, 20]. Therefore it is reasonable to expect our relations to be approximately valid up to a scale $Q_{0}^{2} \approx 1-5 \mathrm{GeV}^{2}[7]$. Thus confirmation of the validity or invalidity of the relation Eq. 21] will be helpful to reveal new content concerning the spin structure of the nucleon.

\section{The light-cone $\mathrm{SU}(6)$ quark-spectator model}

We now discuss the $x$-dependent quark orbital angular momentum distributions $L_{q}(x)$ for the valence $u$ and $d$ quarks in a light-cone $\mathrm{SU}(6)$ quark-spectator model [5]. The unpolarized valence quark distributions $u_{v}(x)$ and $d_{v}(x)$ are given in this model by

$$
\begin{aligned}
& u_{v}(x)=\frac{1}{2} a_{S}(x)+\frac{1}{6} a_{V}(x) ; \\
& d_{v}(x)=\frac{1}{3} a_{V}(x),
\end{aligned}
$$


where $a_{D}(x)$ ( $D=S$ for scalar spectator or $V$ for axial vector spectator) is normalized such that $\int_{0}^{1} d x a_{D}(x)=3$, and it denotes the amplitude for quark $q$ to be scattered while the spectator is in the diquark state $D$. Exact $\mathrm{SU}(6)$ symmetry provides the relation $a_{S}(x)=a_{V}(x)$, which implies the valence flavor symmetry $u_{v}(x)=2 d_{v}(x)$. This gives the prediction $F_{2}^{n}(x) / F_{2}^{p}(x) \geq 2 / 3$ for all $x$, which is ruled out by the experimental observation $F_{2}^{n}(x) / F_{2}^{p}(x)<1 / 2$ for $x \rightarrow 1$. The mass difference between the scalar and vector spectators can reproduce the $u$ and $d$ valence quark asymmetry that accounts for the observed ratio $F_{2}^{n}(x) / F_{2}^{p}(x)$ at large $x$ [5]. This supports the quark-spectator picture of deep inelastic scattering in which the difference between the mass of the scalar and vector spectators is important in order to reproduce the explicit SU(6) symmetry breaking while the bulk SU(6) symmetry of the quark model still holds.

The quark helicity distributions for the $u$ and $d$ quarks can be written as [5]

$$
\begin{aligned}
\Delta u_{v}(x)=u_{v}^{\uparrow}(x)-u_{v}^{\downarrow}(x)=- & \frac{1}{18} a_{V}(x) M_{q}^{V}(x) \\
& +\frac{1}{2} a_{S}(x) M_{q}^{S}(x) ; \\
\Delta d_{v}(x)=d_{v}^{\uparrow}(x)-d_{v}^{\downarrow}(x)=- & \frac{1}{9} a_{V}(x) M_{q}^{V}(x),
\end{aligned}
$$

in which $M_{q}^{S}(x)$ and $M_{q}^{V}(x)$ are the Melosh-Wigner correction factors for the scalar and axial vector spectator-diquark cases. They are obtained by averaging Eq. (5) over $k_{\perp}$ with $\mathcal{M}^{2}=\frac{m_{q}^{2}+k_{\perp}^{2}}{x}+\frac{m_{D}^{2}+k_{\perp}^{2}}{1-x}$, where $m_{D}$ is the mass of the diquark spectator, and are unequal due to unequal spectator masses, which lead to unequal $k_{\perp}$ distributions. From Eq. (22) one gets

$$
\begin{aligned}
& a_{S}(x)=2 u_{v}(x)-d_{v}(x) \\
& a_{V}(x)=3 d_{v}(x)
\end{aligned}
$$

Combining Eqs. (23) and (24) we have

$$
\begin{aligned}
& \Delta u_{v}(x)=\left[u_{v}(x)-\frac{1}{2} d_{v}(x)\right] M_{q}^{S}(x)-\frac{1}{6} d_{v}(x) M_{q}^{V}(x) \\
& \Delta d_{v}(x)=-\frac{1}{3} d_{v}(x) M_{q}^{V}(x) .
\end{aligned}
$$


Thus we arrive at simple relations [5] between the polarized and unpolarized quark distributions for the valence $u$ and $d$ quarks. The relations (25) can be considered as the results of the conventional SU(6) quark model, and which explicitly take into account the Melosh-Wigner rotation effect [3, [- and the flavor asymmetry introduced by the mass difference between the scalar and vector spectators [5].

The extension of relations Eq. (25) to the quark orbital angular momentum distributions $L_{q}(x)$ is straightforward: we can simply replace $M_{q}^{S}(x)$ and $M_{q}^{V}(x)$ by $M_{L}^{S}(x)$ and $M_{L}^{V}(x)$,

$$
\begin{aligned}
& L_{u}(x)=\left[u_{v}(x)-\frac{1}{2} d_{v}(x)\right] M_{L}^{S}(x)-\frac{1}{6} d_{v}(x) L_{L}^{V}(x) \\
& L_{d}(x)=-\frac{1}{3} d_{v}(x) M_{L}^{V}(x) .
\end{aligned}
$$

The $x$-dependent Melosh-Wigner rotation factors $M_{q}^{S}(x)$ and $M_{q}^{V}(x)$ have been calculated [5] and an asymmetry between $M_{q}^{S}(x)$ and $M_{q}^{V}(x)$ was found. Since $\frac{1}{2} M_{q}^{S}(x)+$ $M_{L}^{S}(x)=\frac{1}{2}$ and $\frac{1}{2} M_{q}^{V}(x)+M_{L}^{V}(x)=\frac{1}{2}$, there should be also an asymmetry between $M_{L}^{S}(x)$ and $M_{L}^{V}(x)$ in an opposite direction. The corresponding asymmetry between the Melosh-Wigner rotation factors with the scalar and vector spectators will produce a flavor asymmetry between the averaged Melosh-Wigner rotation factor for the $u$ and $d$ quarks. Contrary to the quark helicity case where the asymmetry causes a reduction of the quark helicity sum compared to the flavor symmetric case, the flavor asymmetry of the Melosh-Wigner rotation factors in the quark orbital angular momentum case produces an increase of the quark orbital angular momentum sum compared to the flavor symmetric case.

The calculated polarization asymmetries $A_{1}^{N}=2 x g_{1}^{N}(x) / F_{2}^{N}(x)$, including the Melosh-Wigner rotation, have been found [5] to be in reasonable agreement with the experimental data, at least for $x \geq 0.1$. A large asymmetry between $M_{q}^{S}(x)$ and $M_{q}^{V}(x)$ leads to a better fit to the data than that obtained from a small asymmetry. Therefore it is reasonable to expect that the calculated $L_{q}(x)$ may lead to predictions close to the real situation. In Fig. (11) we present the calculated $\Delta q(x)$ and $L_{q}(x)$ for the $u$ and $d$ valence quarks. 


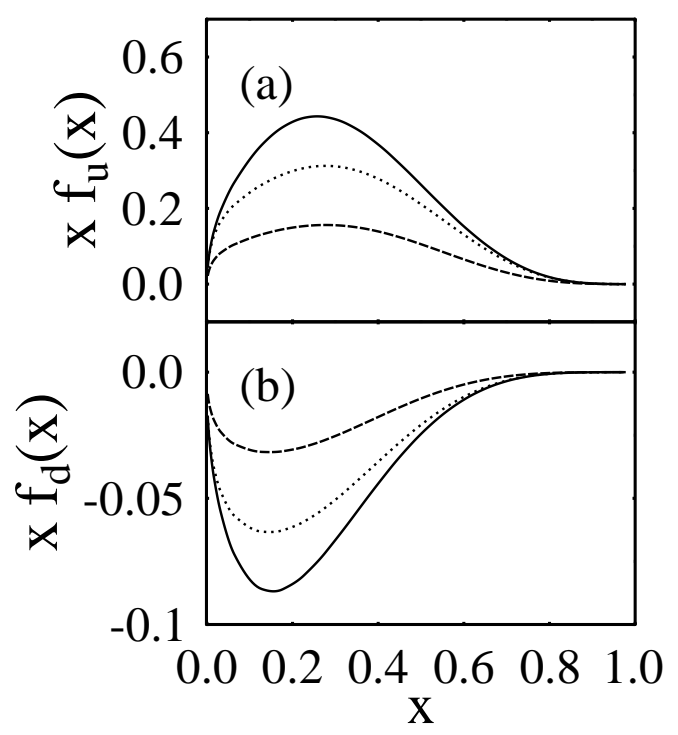

Figure 1: The $x$-dependent quark spin distributions $x \Delta q_{Q M}(x)$ (solid curves), helicity distributions $x \Delta q(x)$ (dotted curves), and orbital angular momentum distributions $x L_{q}(x)$ (dashed curves) in the light-cone $\mathrm{SU}(6)$ quark-spectator model by using Eqs. (25-26), with the Glück-Reya-Vogt parameterization [21] of unpolarized quark distributions as input: (a) for $u$ quarks; (b) for $d$ quarks.

\section{New sum rules for the quark orbital angular momentum}

As we have known, parton sum rules have played important roles in the understanding of the quark-gluon structure of the nucleons. For example, the Gottfried sum rule [22] was proposed initially for the hope to destroy the quark model [23]. However, the confirmation of the roughly validity of the Gottfried sum rule in the early deep inelastic experiments was important for identifying the quantum numbers of partons with those of quarks, thus confirmed the quark model. The recent observation of 
the Gottfried sum rule violation in the refined measurements [24] revealed the flavor asymmetry between the $u$ and $d$ sea quarks [25]. Thus the validity or invalidity of a parton sum rule is useful in order to reveal possible new physics.

New sum rules for the quark tensor charges have been recently proposed [15] based on the relation Eq.(12) [7]. Based on the fact that the anti-quarks in the nucleon sea are likely to be non-polarized from theoretical considerations [26, 27] and experimental evidence [28], a Bjorken-like sum rule for the isovector tensor charge is found

$$
\delta U-\delta D=\frac{1}{2}\left(\frac{g_{A}}{g_{V}}+\frac{5}{3} c_{1}\right)
$$

where $g_{A} / g_{V}$ might be the value from the neutron $\beta$ decay or $g_{A} / g_{V}=6\left(\Gamma^{p}-\Gamma^{n}\right)$ (where the integrals $\Gamma^{p}=\int_{0}^{1} d x g_{1}^{p}(x)$ and $\Gamma^{n}=\int_{0}^{1} d x g_{1}^{n}(x)$ have been measured from polarized DIS experiments), and $c_{1}$ is an unknown correction factor reflecting the deviation from the naive quark model value $\Delta U_{Q M}-\Delta D_{Q M}=5 / 3$. Similarly, there is also a sum rule for the isoscalar tensor charge

$$
\delta U+\delta D=\frac{9}{5}\left(\Gamma^{p}+\Gamma^{n}\right)-\frac{1}{5} \Delta S+\frac{1}{2} c_{2},
$$

where $\Delta S$ is the quark axial charge for the strange flavor and $c_{2}$ is another unknown correction factor reflecting the deviation from the naive quark model value $\Delta U_{Q M}+$ $\Delta D_{Q M}=1$. In fact, $c_{2}$ can be simply taken as the fraction of the proton spin carried by the $u$ and $d$ quarks in the quark model picture.

From the same consideration, we may apply the relation Eq.(19) and arrive at new sum rules for the quark orbital angular momentum $L_{q}=\int_{0}^{1} d x L_{q}(x)$. Corresponding to Eq.(27), we have, for the isovector quark orbital angular momentum,

$$
L_{u}-L_{d}=\frac{1}{2}\left(-\frac{g_{A}}{g_{V}}+\frac{5}{3} c_{1}\right)
$$

and corresponding to Eq.(28), we have, for the isoscalar quark orbital angular momentum,

$$
L_{u}+L_{d}=-\frac{9}{5}\left(\Gamma^{p}+\Gamma^{n}\right)+\frac{1}{5} \Delta S+\frac{1}{2} c_{2} .
$$

We notice that above two sum rules do not require the non-polarized anti-quarks which are required for the validity of the sum rules Eqs.(27) and (28). 
From Eqs. (29) and (30), we can predict the quark orbital angular momenta $L_{u}-L_{d}$ and $L_{u}+L_{d}$ by use of the measurable quantities $\Gamma_{p}, \Gamma_{n}, g_{A} / g_{V}$ and $\Delta S$, and the correction factors $c_{1}$ and $c_{2}$ with limited uncertainties. The quantities $\Gamma^{p}$ and $\Gamma^{n}$ at several different $Q^{2}$ have been measured from polarized DIS experiments [1], 29, 30, 31], and $\Delta S$ has also been extracted from analysis of the polarized DIS data and it might range from about -0.01 [27] to -0.13 [32]. The value of $\Delta S$ from those analysis is sensitive to the assumption of $\mathrm{SU}(3)$ symmetry. It would be better to measure $\Delta S$ from other independent processes and there have been suggestions for this purpose [33, 34]. Nevertheless, we notice that the predicted values of $L_{u}$ and $L_{d}$ are not sensitive to $\Delta S$. The quantities $c_{1}$ and $c_{2}$ reflect the deviation from the naive quark model and their uncertainties can be estimated from known theoretical considerations. For example, from the lattice QCD results for the axial charge $\Delta \Sigma=0.18 \pm 0.10$ [35] and the tensor charge $\delta \Sigma=0.562 \pm 0.088$ [36], we found $\sum \Delta q_{Q M}=0.94 \pm 0.28$ from the relation Eq. (12) and this supports the naive quark picture that the spin of the proton is mostly carried by quarks [7]. Thus $c_{1}$ and $c_{2}$ should be close to the above value for $\sum \Delta q_{Q M}$ from a reasonable consideration. Considering that the gluon may also play an important role in the spin structure of the nucleon [37], we adopt a large uncertainty $c_{2}=0.5 \rightarrow 1$ (which corresponds to a gluon spin contribution in the range $50 \%$ to $0 \%$ ) and $c_{1}=0.7 \rightarrow 1$ in comparison of $c_{2}=0.75 \rightarrow 1$ and $c_{1}=0.9 \rightarrow 1$ adopted in [7]. In case $g_{A} / g_{V}=6\left(\Gamma^{p}-\Gamma^{n}\right)$ is adopted, for $\Gamma^{p}(\mathrm{E} 143)=0.127$ and $\Gamma^{n}(\mathrm{E} 143)=-0.037$ at $\left\langle Q^{2}\right\rangle=3 \mathrm{GeV}^{2}$ [31], we have

$$
\begin{aligned}
& L_{u}-L_{d}=0.09 \rightarrow 0.34 \\
& L_{u}+L_{d}=0.09 \rightarrow 0.34
\end{aligned}
$$

and for $\Gamma^{p}(S M C)=0.136$ and $\Gamma^{n}(S M C)=-0.063$ at $\left\langle Q^{2}\right\rangle=10 \mathrm{GeV}^{2}$ [29], we have

$$
\begin{gathered}
L_{u}-L_{d}=-0.01 \rightarrow 0.24 \\
L_{u}+L_{d}=0.12 \rightarrow 0.37
\end{gathered}
$$

In case the value $g_{A} / g_{V}=1.2573$ from neutron $\beta$ decay is adopted, for the isovector component Eq.(29), we obtain

$$
L_{u}-L_{d}=-0.05 \rightarrow 0.20 .
$$


Combining the results in Eqs.(31), (32) and (33), and taking into account also the uncertainties 0.05 introduced by the data, we obtain

$$
\begin{gathered}
L_{u}-L_{d}=-0.10 \rightarrow 0.39 \\
L_{u}+L_{d}=0.04 \rightarrow 0.42 .
\end{gathered}
$$

Progress in the precision of the data and in the knowledge of the correction factors can further constrain the results.

In fact, we can use the two sets of sum rules, Eqs.(27) and (28) and Eqs.(29) and (30), as two independent measures of the quantities $c_{1}$ and $c_{2}$ which might be considered as model quantities with no universal significance. From one set of above sum rules one can measure the quantities $c_{1}$ and $c_{2}$, and then use them as inputs to another set of sum rules to make the prediction. However, by use of the relation Eq.(21) we may have a new sum rule which directly connects the quark orbital angular momentum to the quark axial and tensor charges

$$
L_{q}=\delta Q-\Delta q
$$

which is much simple. From the lattice QCD calculations we have the axial charge $\Delta \Sigma=0.18 \pm 0.10$ [35] and the tensor charge $\delta \Sigma=0.562 \pm 0.088$ [36], thus the total quark orbital angular momentum from the new sum rule Eq.(35) should be

$$
\Sigma L_{q}=\delta \Sigma-\Delta \Sigma=0.38 \pm 0.13
$$

which implies that $76 \pm 26 \%$ of the proton spin is due to the quark relativistic orbital motions from the known lattice results for the nucleon axial and tensor charges.

In similar to the relation $\Delta q_{Q M}(x)+\Delta q(x)=2 \delta(x)$ discussed in Ref. [7, 15], the relations $\frac{1}{2} \Delta q(x)+L_{q}(x)=\frac{1}{2} \Delta q_{Q M}(x)$ and $\delta q(x)+L_{q}(x)=\Delta q(x)$ are valid for each flavor quark, likewise for each flavor anti-quark. The sum rule Eq.(35) will require non-polarized anti-quarks due to the charge conjugation properties of the tensor current. However, as have been pointed out previously [7, 15], the nonpolarization of anti-quarks has been clearly predicted in a broken-U(3) version of the chiral quark model [26] and in the meson-baryon fluctuation model [27]. There has been an explicit measurement of the helicity distributions for the individual $u$ 
and $d$ valence and sea quarks by the Spin Muon Collaboration (SMC) [28]. The refined SMC measurement of the helicity distributions for the $u$ and $d$ anti-quarks are consistent with zero in agreement with the above predictions. Therefore the small anti-quark polarization can be considered as a fact supported by both experimental measurement [28] and theoretical considerations [26, 27]. We need to indicate that we have neglected the contributions from anti-quarks, gluons, $Q^{2}$ dependence due to higher twist effects, and different evolution behaviors of quark distributions et al. in the above analysis. In principle the corrections due to these sources can be further taken into account from the theoretical and experimental progress and they should be topics for further study.

\section{Summary}

In summary, we performed an analysis of the quark angular momentum in a light-cone representation by taking into account the effect due to the Melosh-Wigner rotation and found a similar relativistic correction factor connecting the quark orbital angular momentum to the quark model spin distribution: $L_{q}(x)=<M_{L}(x)>\Delta q_{Q M}(x)$. The quark orbital angular momentum $L_{q}(x)$ and the quark helicity distribution $\Delta q(x)$ are connected to the quark model spin distribution $\Delta q_{Q M}(x)$ by a relation: $\frac{1}{2} \Delta q(x)+L_{q}(x)=\frac{1}{2} \Delta q_{Q M}(x)$, which means that one can decompose the quark model spin contribution $\Delta q_{Q M}(x)$ by a quark helicity term $\Delta q(x)$ plus an orbital angular momentum term $L_{q}(x)$. There is also a new relation connecting the quark orbital angular momentum with the measurable quark helicity distribution and transversity distribution: $\Delta q(x)+L_{q}(x)=\delta q(x)$, from which we have a new sum rule connecting the quark orbital angular momentum with the nucleon axial and tensor charges. Two other new sum rules for the quark orbital angular momentum are proposed and the values for the isovector and isoscalar quark orbital angular momenta $L_{u}-L_{d}$ and $L_{u}+L_{d}$ are estimated from the measured quantities $\Gamma^{p}, \Gamma^{n}, g_{A} / g_{V}$ and $\Delta S$, and two model correction factors with limited uncertainties. We also calculated the $x$-dependent quark orbital angular momentum distributions for the $u$ and $d$ valence quarks in a light-cone $\mathrm{SU}(6)$ quark-spectator model. 
Acknowledgments: We would like to thank S.J. Brodsky and J. Soffer for helpful discussions. This work is partially supported by National Natural Science Foundation of China under Grant No. 19605006, Fondecyt (Chile) under grant 1960536, by a Cátedra Presidencial (Chile), and by Fundación Andes (Chile). 


\section{References}

[1] EM Collab., J. Ashman et al., Phys. Lett. B 206, 364 (1988); Nucl. Phys. B 328, 1 (1989).

[2] For reviews, see, e.g.,

H.-Y. Cheng, Int. J. Mod. Phys. A 11, 5109 (1996);

G.P. Ramsey, Prog. Part. Nucl. Phys. 39, 599 (1997).

[3] B.-Q. Ma, J. Phys. G 17, L53 (1991);

B.-Q. Ma and Q.-R. Zhang, Z. Phys. C 58, 479 (1993).

[4] S.J. Brodsky and F. Schlumpf, Phys. Lett. B 329, 111 (1994).

[5] B.-Q. Ma, Phys. Lett. B 375, 320 (1996);

B.-Q. Ma and A. Schäfer, Phys. Lett. B 378, 307 (1996).

[6] I. Schmidt and J. Soffer, Phys. Lett. B 407, 331 (1997).

[7] B.-Q. Ma, I. Schmidt, and J. Soffer, hep-ph/9710247, to be published in Phys. Lett. B.

[8] D. Qing, X.-S. Chen, and F. Wang, hep-ph/9802425.

[9] P. Zavada, Phys. Rev. D 56, 5834 (1997); hep-ph/9803443.

[10] H.J. Melosh, Phys. Rev. D 9, 1095 (1974);

E. Wigner, Ann. Math. 40, 149 (1939).

[11] See, e.g., S.J. Brodsky, H-C. Pauli, and S.S. Pinsky, Phys. Rep. 301, 299 (1998), and references therein.

[12] See, e.g.,

L.A. Kondratyuk and M.V. Terent'ev, Yad.Fiz 31, 1087 (1980) [ Sov. J. Nucl. Phys. 31, 561 (1980)]; 
P.L. Chung, F. Coester, B.D. Keister, and W.N. Polyzou, Phys. Rev. C 37, 2000 (1988);

H.J. Weber, Ann. Phys. (N.Y.) 207, 417 (1991);

W. Jaus, Phys. Rev. D 41, 3394 (1990); D 44, 2851 (1991);

P.L. Chung and F. Coester, Phys. Rev. D 44, 229 (1991);

B.-Q.Ma, Z. Phys. A 345, 325 (1993);

F. Schlumpf, Phys. Rev. D 48, 4478 (1993);

F. Schlumpf and S.J. Brodsky, Phys. Lett. 360, 1 (1995).

T. Huang, B.-Q.Ma, and Q.-X. Shen, Phys. Rev. D 49, 1490 (1994);

B.-Q. Ma and T. Huang, J. Phys. G 21, 765 (1995);

F.-G. Cao, J. Cao, T. Huang, and B.-Q. Ma, Phys. Rev. D 55, 7107 (1997).

[13] See, e.g., S. J. Brodsky and G. P. Lepage, in Perturbative Quantum Chromodynamics, edited by A. H. Mueller (Singapore, World Scientific, 1989), p. 93;

S. J. Brodsky, T. Huang, and G. P. Lepage, in Particles and Fields-2, Proceedings of the Banff Summer Institute, Banff, Alberta, 1981, edited by A. Z. Capri and A. N. Kamal (Plenum, New York,1983), p. 143; G. P. Lepage, S. J. Brodsky, T. Huang, and P. B. Mackenize, ibid., p. 83.

[14] G.P. Lepage and S.J. Brodsky, Phys. Rev. D 22, 2157 (1980);

W. Konen and H.J. Weber, Phys. Rev. D 41, 2201 (1990).

[15] B.-Q. Ma and I. Schmidt, hep-ph/9711326, to be published in J. Phys. G.

[16] L.M. Sehgal, Phys. Rev. D 10, 1663 (1974).

[17] See, e.g., X. Song, hep-ph/9801332; P. Hoodbhoy, X. Ji, and W. Lu, hep$\mathrm{ph} / 9804337$; and references therein.

[18] F. Martin, Phys. Rev. D 19, 1382 (1979);

M. Glück, E. Reya, and W. Vogelsang, Nucl. Phys. B 329, 347 (1990). 
[19] S.J. Brodsky and I. Schmidt, Phys. Lett. B 234, 144 (1990);

P. Hoyer and D.P. Roy, Phys. Lett. 410, 63 (1997).

[20] F. Schlumpf, J. Phys. G 20, 237 (1994);

See also, F.Schlumpf, Ph.D. thesis, University of Zurich, 1992.

[21] M. Glück, E. Reya, and A. Vogt, Z. Phys. C 67, 433 (1995). We use the LO set at $Q^{2}=5 \mathrm{GeV}^{2}$ in the calculations.

[22] K. Gottfried, Phys. Rev. Lett. 18, 1174 (1967).

[23] Proc. 1967 International Symposium on Electron and Photon Interactions at High Energy, Stanford, California, September 5-9, 167.

[24] NM Collab., P. Amaudruz et al., Phys. Rev. Lett. 66, 2712 (1991);

M. Arneodo et al., Phys. Rev. D 50, R1 (1994).

[25] G. Preparata, P.G. Ratcliffe, and J. Soffer, Phys. Rev. Lett. 66, 687 (1991).

[26] T.P. Cheng and L.F. Li, Phys. Lett. B 366, 365 (1996).

[27] S.J. Brodsky and B.-Q. Ma, Phys. Lett. B 381, 317 (1996).

[28] SM Collab., B. Adeva et al., Phys. Lett. B 369, 93 (1996); for most recent refined results, see, Phys. Lett. 420, 180 (1998).

[29] SM Collab., B. Adeva et al., Phys. Lett. B 302, 533 (1993); B 357, 248 (1995);

D. Adams et al., ibid. B 329, 399 (1994); B 339, 332(E) (1994).

[30] E142 Collab., P.L. Anthony et al., Phys. Rev. Lett. 71, 959 (1993).

[31] E143 Collab., P.L. Anthony et al., Phys. Rev. Lett. 74, 346 (1995); K. Abe et al., ibid. 75, 25 (1995).

[32] J. Ellis and M. Karliner, Phys. Lett. B 341, 397 (1995). 
[33] W. Lu and B. -Q. Ma, Phys. Lett. B 357, 419 (1995);

W. Lu, Phys. Lett. B 373, 223 (1996).

[34] J. Ellis, D. Kharzeev, and A. Kotzinian, Z. Phys. C 69, 467 (1995).

[35] M. Fukugita, Y. Kuramashi, M. Okawa, and A. Ukawa, Phys. Rev. Lett. 75, $2092(1995)$.

[36] S. Aoki, M. Doui, T. Hatsuda, and Y. Kuramashi, Phys. Rev. D 56, 433 (1997).

[37] See, e.g., G. Altarelli and G.G. Ross, Phys. Lett. B 212, 391 (1988);

R.D. Carlitz, J.C. Collins, and A.H. Mueller, Phys. Lett. 214, 229 (1988);

A.V. Efremov, J. Soffer, and O.V. Teryaev, Nucl. Phys. B 346, 97 (1990).

For most recent discussions, see, e.g., J. Soffer and O.V. Teryaev, Phys. Lett. 419, 400 (1998); V. Barone, T. Calarco, and A. Drago, hep-ph/9801281; and references therein. 